\title{
Переход от экспоненциального к линейному возрастанию плотности энергии спектральной компоненты пикосекундного стимулированного излучения GaAs при насыщении усиления
}

\author{
(C) Н.Н. Агеева, И.Л. Броневой , Д.Н. Забегаев, А.Н. Кривоносов \\ Институт радиотехники и электроники им. В.А. Котельникова Российской академии наук, \\ 125009 Москва, Россия \\ I E-mail: bil@cplire.ru
}

Поступила в Редакцию 1 декабря 2021 г.

В окончательной редакции 25 декабря 2021 г.

Принята к публикации 27 декабря 2021 г.

B начале мощной оптической пикосекундной накачки слоя GaAs гетероструктуры $\mathrm{Al}_{x} \mathrm{Ga}_{1-x} \mathrm{As}-\mathrm{GaAs}-\mathrm{Al}_{x} \mathrm{Ga}_{1-x} \mathrm{As}$ в нем возникает стимулированное пикосекундное излучение. Возрастание со временем плотности энергии каждой спектральной компоненты излучения в ее активной среде происходит экспоненциально до насыщения усиления, далее возрастание линейное. В настоящей работе экспериментально определено, в зависимости от каких параметров спектральной компоненты излучения и по какому закону меняется: (а) время (отсчитываемое от начала стимулированного излучения), через которое происходит переход от экспоненциального возрастания к линейному; (б) плотность энергии компоненты в „момент“ перехода; (в) коэффициент усиления на этапе линейного возрастания плотности энергии. В Заключении суммируются явления при насыщении усиления, обнаруженные в наших работах.

Ключевые слова: стимулированное пикосекундное излучение, арсенид галлия, спектральные компоненты излучения, характерное время релаксации излучения, насыщение усиления, энергетический транспорт носителей заряда, скорость вынужденной рекомбинации.

DOI: 10.21883/FTP.2022.04.52194.9781

\section{1. Введение}

Через несколько пикосекунд после начала мощной оптической накачки тонкого слоя GaAs, входящего в состав гетероструктуры $\mathrm{Al}_{x} \mathrm{Ga}_{1-x} \mathrm{As}-\mathrm{GaAs}-\mathrm{Al}_{x} \mathrm{Ga}_{1-x} \mathrm{As}$, в слое возникает мощное стимулированное пикосекундное излучение. Для краткости будем называть его $s$-излучение. В работе [1] показано, что сначала интенсивность спектральной компоненты $s$-излучения, вышедшего из гетероструктуры, растет экспоненциально со временем. Это аналогично ненасыщенному усилению „слабого“ света по закону Бугера. Через некоторое время происходил переход от экспоненциального к линейному возрастанию интенсивности компоненты со временем. Подобное изменение характера возрастания, но в несколько других условиях, предсказывалось теоретически при насыщении усиления [2,3]. Последнее означает режим, когда излучение уже влияет на собственное усиление. Установленный в [1] коэффициент линейного возрастания ясно указывал, что существенную роль в линейном возрастании выполняет транспорт накаченных носителей в энергетическом пространстве. На этот транспорт в свою очередь влияет взаимодействие носителей заряда с $s$-излучением.

В настоящей работе исследуются параметры и закономерности перехода от экспоненциального к линейному возрастанию плотности энергии спектральной компоненты $s$-излучения в ее активной среде. Это позволило установить экспериментально условия перехода от режима ненасыщенного усиления к режиму насыщения усиления. Выяснено изменение коэффициента усиления при линейном возрастании плотности энергии компоненты. Работа восполнила не достававшее звено, чтобы вместе с ранее выполненными исследованиями можно было представить, в первом приближении, эволюцию „каждой“ спектральной компоненты $s$-излучения на всех ее этапах.

\section{2. Экспериментальные результаты}

$S$-излучение генерировалось в слое GaAs гетероструктуры $\mathrm{Al}_{0.22} \mathrm{Ga}_{0.78} \mathrm{As}-\mathrm{GaAs}-\mathrm{Al}_{0.4} \mathrm{Ga}_{0.6} \mathrm{As}$ при его накачке световым импульсом длительностью (FWHM) $T_{\mathrm{ex}} \approx 10$ пс, диаметром луча в местоположении образца $(\mathrm{FWHM}) \quad D_{\mathrm{ex}}=0.5 \mathrm{MM}$ и с энергией фотона $\hbar \omega_{\mathrm{ex}} \approx 1.512$ эВ. Слои $\mathrm{Al}_{x} \mathrm{Ga}_{1-x} \mathrm{As}$ прозрачны для света, используемого в эксперименте. На поверхности гетероструктуры (исключая поверхность торцов) нанесено антиотражающее покрытие. Измерялась часть $s$-излучения GaAs, выходившая из накачанной области слоя и распространявшаяся внутри телесного угла $1.2 \cdot 10^{-2}$ стерадиан с осью, ортогональной поверхности гетероструктуры. Эта часть излучения была выбрана для измерений потому, что выходит прямо из активной области, не изменяясь из-за поглощения в пассивной области и отражения от торцов образца. В работе [4] суммированы доказательства того, что указанная часть и более интенсивное излучение, выходящее из торца 


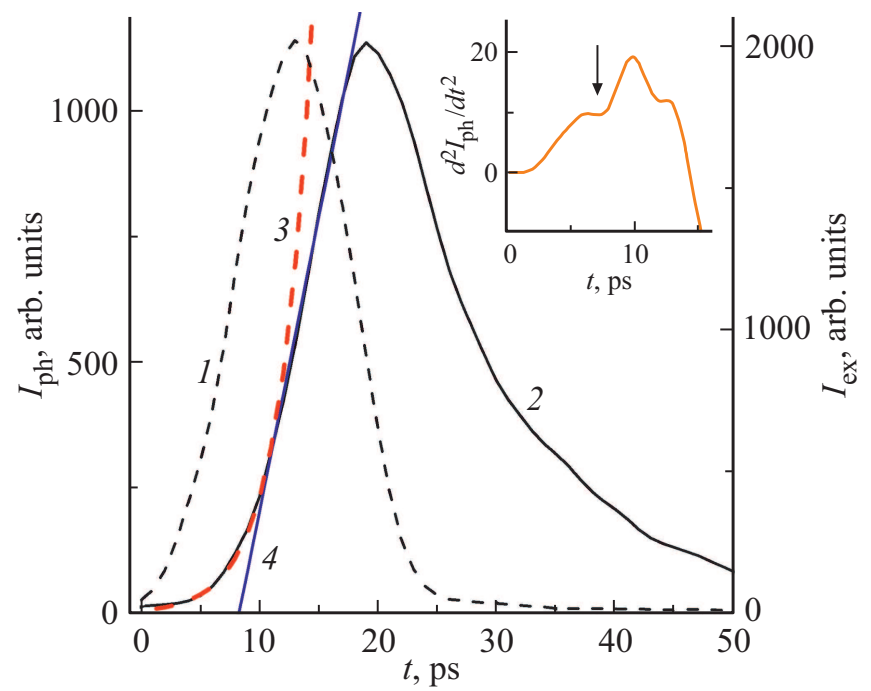

Рис. 1. Хронограммы импульсов: накачки $I_{\mathrm{ex}}(t)(1)$ и $s$-компоненты $I_{\mathrm{ph}}(t)$, энергия фотона которой $\hbar \omega=1.396$ эВ (2). Кривые 3 и 4 - соответственно экспоненциальная и линейная касательные к хронограмме $s$-компоненты. На вставке зависимость $d^{2} I_{\mathrm{ph}} / d t^{2}=f(t)$ для части фронта $s$-компоненты.

образца, составляют одно и то же стимулированное излучение.

Для краткости импульсы спектральных компонент $s$-излучения будем называть $s$-компонентами. В настоящей работе мы опираемся на измерения в реальном времени огибающих $s$-компонент. Измерение огибающих описано, например, в [4] ${ }^{1}$. Результаты измерения огибающей импульса накачки и огибающей $s$-компоненты, представленные в графическом виде, будем называть хронограммами и обозначать $I_{\mathrm{ex}}(t)$ и $I_{\mathrm{ph}}(t)$, где $I_{\mathrm{ex}}$ и $I_{\mathrm{ph}}$ - интенсивности соответственно света накачки и $s$-компоненты на приемной площадке фотоприемника, $t$ - время (рис. 1 , кривые 1 и 2). На том же рисунке кривые 3 и 4 - это соответственно экспоненциальная и линейная касательные к хронограмме $s$-компоненты. В области перекрытия кривых 3 и 4 происходит переход от экспоненциального к линейному возрастанию интенсивности $s$-компоненты. Для краткости будем называть его $g$-переход.

Интервал времени, в которое происходит $g$-переход, и его середина представлены отрезком прямой и точкой соответственно на графике $t_{\mathrm{th}}(\hbar \omega)$, где $\hbar \omega-$ энергия фотона $s$-компоненты (рис. 2). Аналогично интервал интенсивности $I_{\mathrm{ph}}$, в котором происходит $g$-переход, и его середина представлены отрезком прямой и точкой соответственно на графике $I_{\mathrm{th}}(\hbar \omega)$ (рис. 3). Диаметр

\footnotetext{
${ }^{1}$ Мы вынуждены в каждой статье пояснять, что ссылки на наши предыдущие работы приводятся вынужденно, а не для саморекламы. Заменить их ссылками на работы других авторов не удается, возможно, вследствие специфичности спектрофотохронометрического комплекса, на котором велись исследования. Перечень наших работ, посвященных исследованию $s$-излучения, приведен, в частности, на сайте нашего института http://www.cplire.ru/rus/bil/index.html
}

$L$ активной области, в которой генерируется $s$-компонента, был определен в работе [5]. Спектр $L(\hbar \omega)$ представлен на рис. 4, кривой 1. На том же рисунке

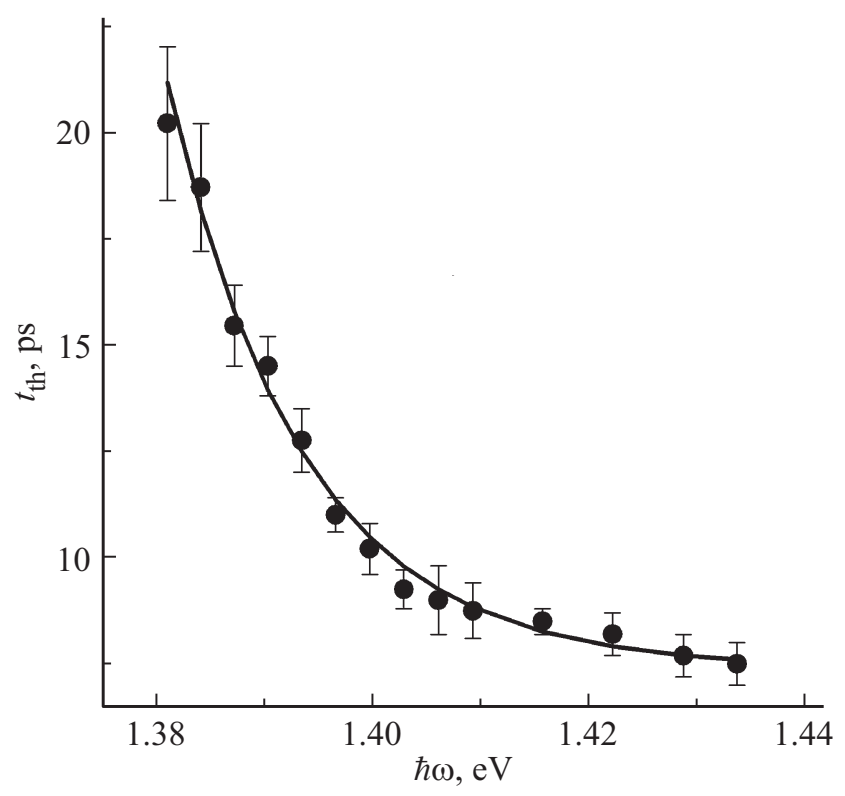

Рис. 2. Вертикальные отрезки показывают интервал времени от начала до конца $g$-перехода $s$-компоненты с энергией фотона $\hbar \omega$, символ - - момент времени $t_{\text {th }}$, на который приходится середина интервала. Сплошная кривая построена по формуле (1).

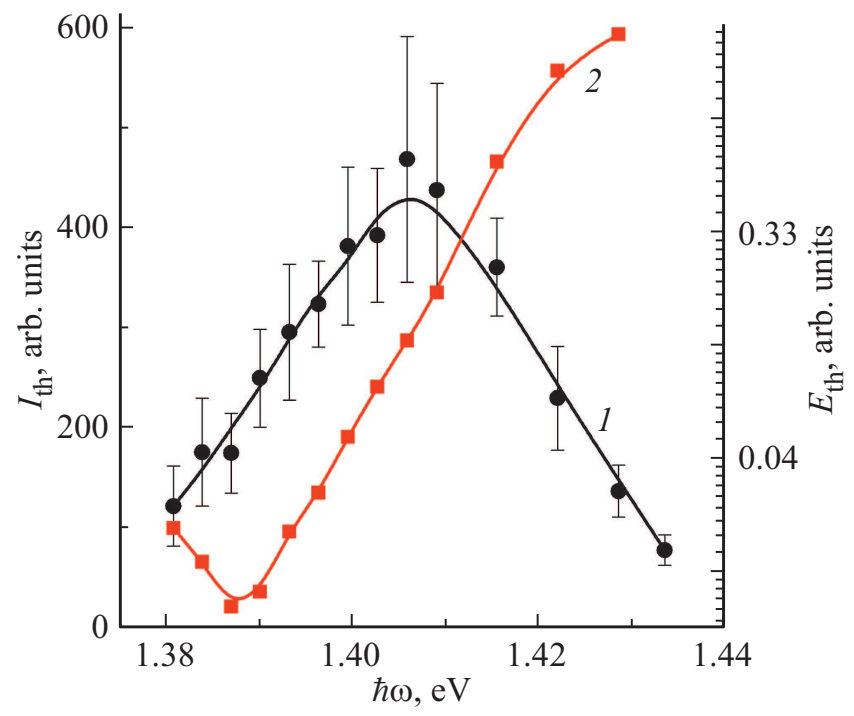

Рис. 3. 1 - интервал интенсивности $s$-компоненты от начала до конца $g$-перехода (вертикальные отрезки) и интенсивность $I_{\text {th }}$ в середине интервала (символ •) в функции от энергии фотона $\hbar \omega s$-компоненты (сказанное относится к интенсивности, непосредственно измеренной фотоприемником). 2 - средняя по активной среде плотность энергии $E_{\text {th }}$ $s$-компоненты во время $g$-перехода, тоже в функции от $\hbar \omega$. Сплошные линии здесь и на следующем рисунке проведены для наглядности. 

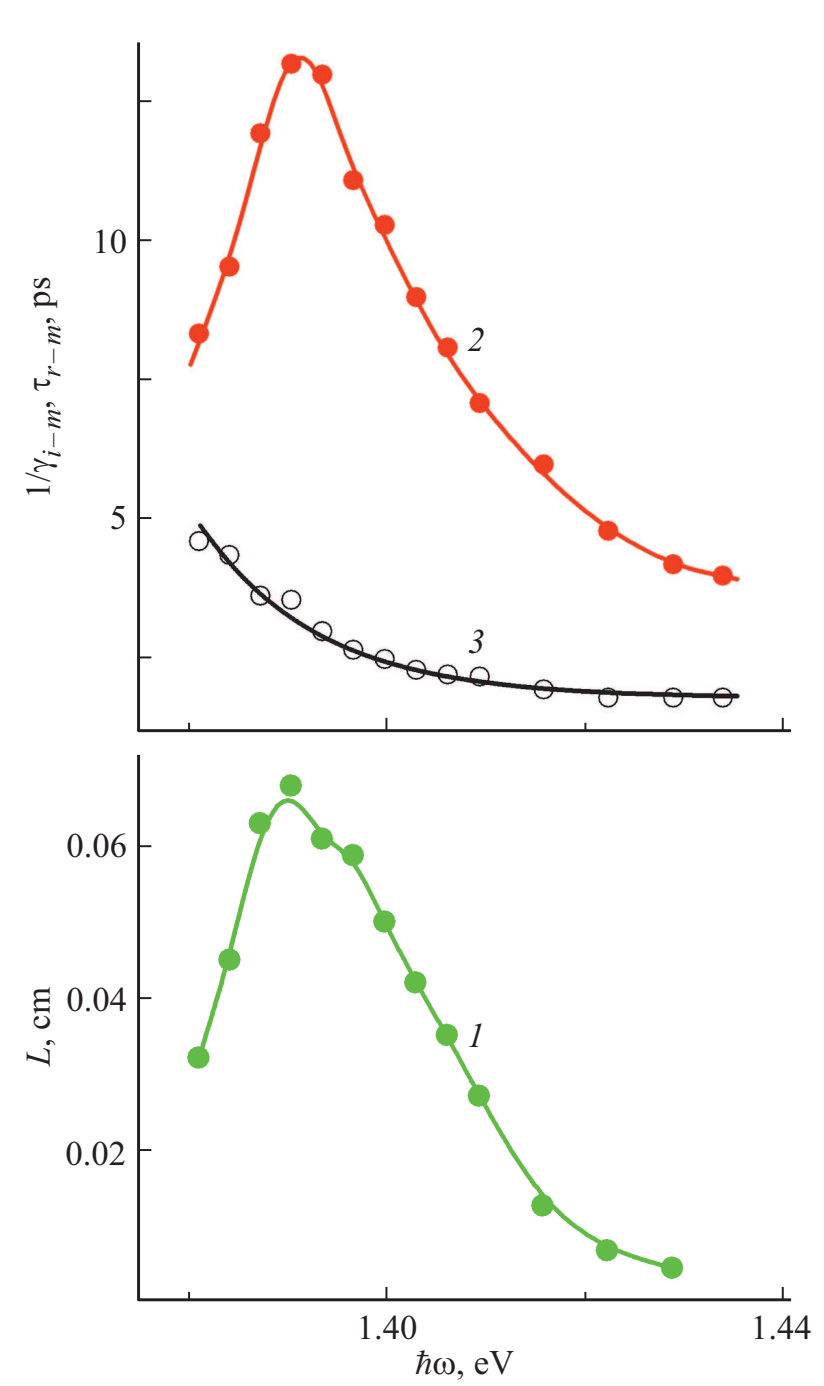

Pис. 4. Спектры: диаметра $L$ активной области, в которой генерируется $s$-компонента $(1)$; характерного времени релаксации $\tau_{r-m}(2)$ и обратного коэффициента ненасыщенного усиления $1 / \gamma_{i-m}(3) s$-компоненты. (Цветной вариант рисунка представлен в электронной версии статьи).

представлены требуемые далее спектры: характерного времени релаксации интенсивности $I_{\mathrm{ph}}$ на спаде $s$-компоненты - $\tau_{r-m}(\hbar \omega)$ из работы [6] (кривая 2) и обратного коэффициента ненасыщенного экспоненциального усиления $-1 / \gamma_{i-m}=f(\hbar \omega)$ из работы [1] (кривая 3). Термин „ненасыщенное“ в данном случае означает, что излучение еще не влияет на коэффициент усиления. Далее мы обсудим, какие закономерности $g$-перехода следуют из вышеперечисленных экспериментальных результатов.

\section{3. Универсальное условие $g$-перехода}

Обнаруженное возрастание времени $t_{\text {th }}$ при уменышении $\hbar \omega$ (рис. 2) имеет экспоненциальный характер и может быть аппроксимировано эмпирической формулой

$$
t_{\mathrm{th}}=7.4+0.6 \cdot \exp (-80 \cdot(\hbar \omega-1.42)) \text {, }
$$

где $t_{\text {th }}-$ в пс, $\hbar \omega-$ в эВ, 1.42 эВ $\approx E_{g 0}-$ ширина запрещенной зоны невозбужденного GaAs. Сопоставление форм спектров $t_{\text {th }}(\hbar \omega)$ и $1 / \gamma_{i-m}=f(\hbar \omega)$ позволят предположить связь $t_{\text {th }}$ и $1 / \gamma_{i-m}$. Действительно, как обнаруживается из графика на рис. 5, между этими параметрами существует линейная зависимость

$$
t_{\mathrm{th}}=4.2 / \gamma_{i-m}
$$

Из нее следует главный вывод этой работы: $g$-переход происходит, когда будет выполнено универсальное условие

$$
\gamma_{i-m} \cdot t_{\mathrm{th}}=4.2
$$

Подобное (3) по сути условие перехода от экспоненциального к линейному возрастанию, но только в одномерной, стационарно и однородно накачиваемой активной среде, было теоретически предсказано в упоминавшейся выше работе [2]:

$$
g_{h} \cdot l \sim 10,
$$

где $g_{h}-$ коэффициент ненасыщенного усиления излучения в пространстве активной среды, $l-$ расстояние, пройденное излучением однократно в активной среде. Условие (4) - это один из результатов решения уравнений насыщения для беззеркальных лазерных усилителей в указанной работе. Корректно сравнить количественно условия (3) и (4) затруднительно, в частности, из-за следующего отличия. В нашем случае излучение генерируется в динамическом режиме, при пикосекундной

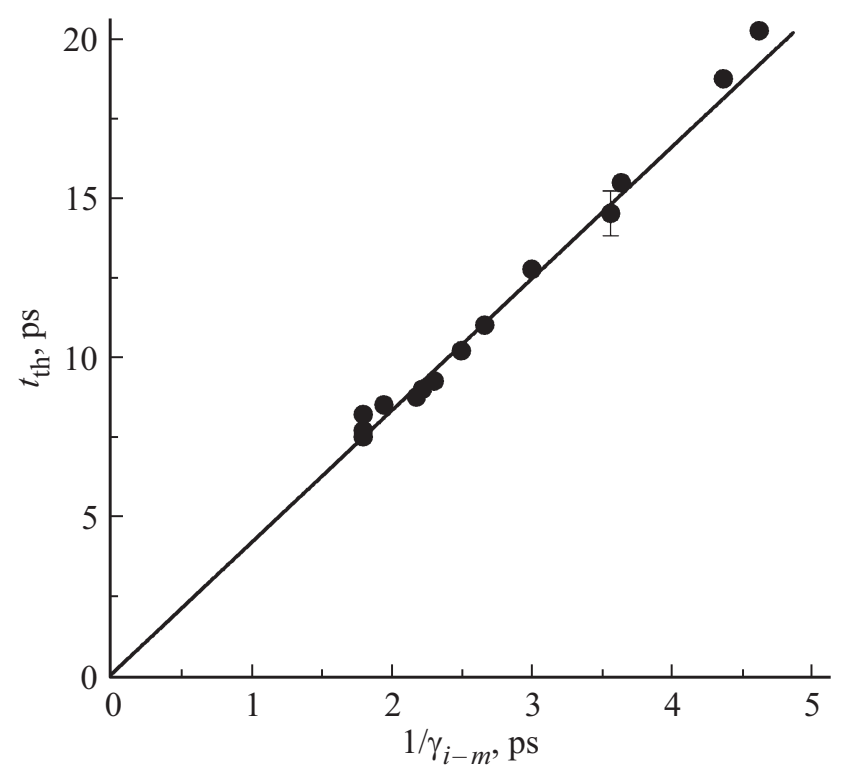

Рис. 5. Зависимость момента времени $t_{\text {th }}$ в середине $g$-перехода от обратной величины коэффициента ненасыщенного усиления $1 / \gamma_{i-m}$. Сплошная прямая построена по формуле (2). 
накачке, в активной среде волноводной гетероструктуры и, как допустимо представить, парциальные волны, движущиеся навстречу друг другу, формируют почти стоячие волны [7]. Как грубое приближение, только чтобы сравнить произведения (3) и (4) по порядку величины, можно принять соотношение

$$
\gamma_{i-m} \approx g_{h} \cdot c / 2 \chi
$$

где $\chi=3.6$ - показатель преломления, $c-$ скорость света в вакууме, коэффициент 2 связан с тем, что усиление происходит только в местоположении пучностей почти стоячей волны. Принимая также, что $l=c \cdot t_{\mathrm{th}} / \chi$ и $g_{h}$ определяется из выражения (5), после подстановки их в (4), и учитывая (3), получаем $g_{h} \cdot l \approx 8.4$. Такое значение $g_{h} l$ не противоречит условию (4), указывающему только порядок величины этого произведения. Таким образом, мы экспериментально получили универсальное условие (4) перехода от экспоненциального к линейному возрастанию во времени (или от режима ненасыщенного усиления к режиму насыщения усиления) стимулированного излучения, генерируемого в волноводной полупроводниковой гетероструктуре. Это условие не противоречит предсказанному ранее теоретически для усиления в пространстве.

\section{4. Спектр пороговой плотности энергии $s$-компонент}

Учитывая, что в соответствии с круглым фокусным пятном луча накачки активная среда в волноводной гетероструктуре для каждой $s$-компоненты по форме похожа на диск, предположено следующее. Спектральная компонента в активной области подобна цилиндрической волне, частично стоячей (можно сказать и частично бегущей). При таком представлении излучения корректнее считать, что $I_{\mathrm{ph}}(t) \sim E_{s}(t)$, где $E_{s}$ - энергия $s$-компоненты в активной среде в момент времени $t$. Тогда из спектра интенсивности $I_{\mathrm{th}}(\hbar \omega)$ во время $g$-перехода и спектра диаметра активной области $L(\hbar \omega) s$-компоненты получаем спектр средней по активной среде плотности энергии $s$-компоненты во время $g$-перехода $E_{\text {th }}=k \cdot I_{\text {th }} / L^{2}=f(\hbar \omega)$, где $k=$ const. Обоснованность подобного рода процедуры очевидна еще и из пояснений в работе [5]. Спектр $E_{\text {th }}(\hbar \omega)$ представлен в полулогарифмическом масштабе (для большей ясности мелкомасштабных особенностей) на рис. 3. Обращает на себя внимание то, что такой график качественно антикоррелирует с графиком спектра характерного времени релаксации $s$-компоненты $\tau_{r-m}=f(\hbar \omega)$, представленным на рис. 4. Точнее, как выяснилось, плотность энергии $E_{\mathrm{th}}$ связана с суммой $\left(\tau_{r-m}+1 / \gamma_{i-m}\right)$ зависимостью

$$
E_{\mathrm{th}}=0.045+250 \cdot \exp \left(-\left(1 / \gamma_{i-m}+\tau_{r-m}\right) / 1.6\right)
$$

график которой представлен сплошной кривой на рис. 6 . Этот график дан в полулогарифмическом масштабе

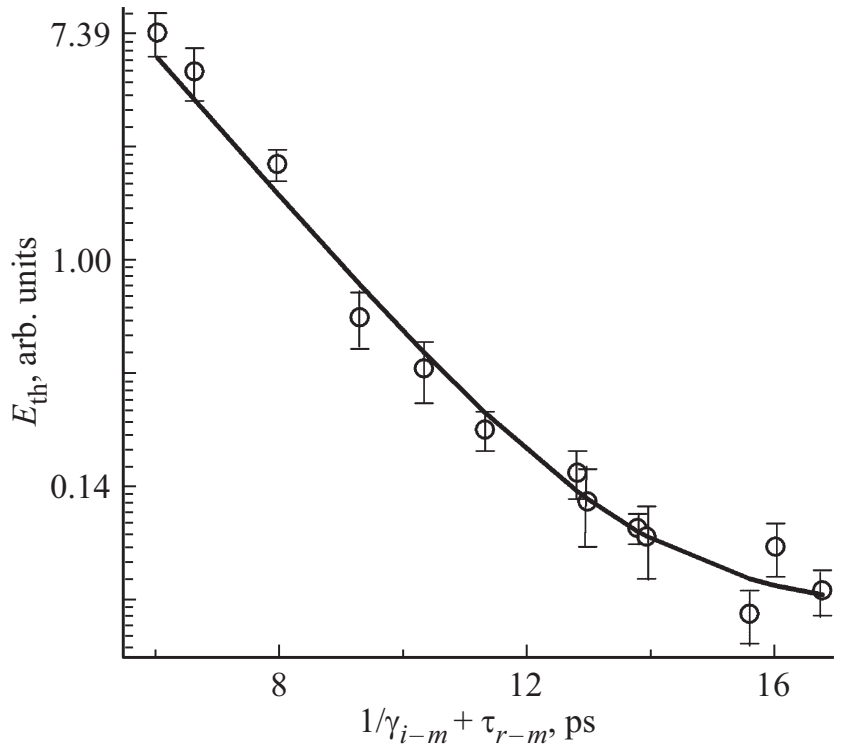

Рис. 6. Плотность энергии $E_{\text {th }} s$-компоненты во время $g$-перехода, средняя по активной среде, в функции от суммы $\left(1 / \gamma_{i-m}+\tau_{r-m}\right)$.

опять же для большей ясности его мелкомасштабных особенностей и их соответствия аппроксимации (6). Отклонение экспериментальных точек от кривой на рис. 6, возможно, вызвано: (a) конечной протяженностью $g$-перехода; (б) суммированием погрешностей измерений четырех физических параметров, фактически связанных зависимостью (6), т. е. $I_{\mathrm{th}}, L, \gamma_{i-m}$ и $\tau_{r-m}$. Зависимость (6) подтверждается и тем, что экспериментальные точки $E_{\mathrm{th}}$, относящиеся к разным $\hbar \omega, \tau_{r-m}$ и $\gamma_{i-m}$, практически совпадают на графике $E_{\mathrm{th}}=f\left(\tau_{r-m}+1 / \gamma_{i-m}\right)$ при одинаковом значении суммы $\left(\tau_{r-m}+1 / \gamma_{i-m}\right)$.

\section{5. Обсуждение установленных закономерностей}

В энергетическом пространстве происходит транспорт накачанных носителей (ЭТН) к экстремумам зон проводимости и валентной, откуда они потом рекомбинируют. В области экстремумов ЭТН делится на части. Обозначим МТН (микротранспорт носителей) как часть ЭТН, заполняющую носителями (электронами и дырками) пары одночастичных квантовых состояний, связанных прямым оптическим переходом с фиксированной энергией $\hbar \omega$ фотона. Одно состояние из каждой пары находится на энергетическом $(c$-) уровне в зоне проводимости, другое - на энергетическом $(v$-) уровне в валентной зоне. Обозначим вероятность заполнения этих состояний $f_{c}$ и $f_{v}$ соответственно. До наступления $g$-перехода спектр коэффициента усиления неизменен, $\gamma_{i-m}=$ const при фиксированной $\hbar \omega$ [1]. Поэтому в первом приближении можно представить, что скорость 
вынужденной рекомбинации носителей с каждой пары оптически связанных $c v$-уровней и МТН на эту пару уровней находятся в динамическом равновесии, возрастая экспоненциально со временем. Это режим ненасыщенного усиления (режим-1). Наступает момент, когда скорость рекомбинации возросла настолько, что МТН не сможет далее компенсировать рекомбинацию носителей, доставленных им на пару $c v$-уровней. Начинается уменьшение инверсии населенности. Возникает провал в спектре усиления, обнаруженный экспериментально в работе [8]. Далее на фронте энергия $s$-компоненты в активной среде растет уже линейно со временем

$$
E_{s}=E_{\mathrm{th}}\left[1+\gamma_{L}\left(t-t_{\mathrm{th}}\right)\right] \text {. }
$$

Это вытекает из пропорциональности $I_{\mathrm{ph}}(t) \sim E_{s}(t)$ и эмпирических формул [1]

$$
\begin{gathered}
I_{\mathrm{ph}}=I_{\mathrm{th}}\left[1+\gamma_{L}\left(t-t_{\mathrm{th}}\right)\right], \\
\gamma_{L}(\hbar \omega) \approx \gamma_{i-m}(\hbar \omega)+1 / \tau_{r-m}(\hbar \omega) .
\end{gathered}
$$

Изменение света в активной среде обычно описывают с помощью дифференциального коэффициента усиления [3], в нашем случае определяемого выражением

$$
\gamma=\left(1 / E_{s}\right) \cdot\left(d E_{s} / d t\right)
$$

Тогда из (7), (10) следует, что коэффициент усиления $\gamma$ должен в процессе $g$-перехода достичь величины $\gamma_{L}$, а далее, пока выполняется (7), уменьшаться со временем:

$$
\gamma=\gamma_{L}\left[1+\gamma_{L}\left(t-t_{\mathrm{th}}\right)\right]^{-1} \text {. }
$$

Попробуем предположить, как, согласно эксперименту, коэффициент $\gamma$ во время $g$-перехода увеличивается от $\gamma_{i-m}$ до $\gamma_{L}$. Согласно сказанному несколько выше, в какой-то момент скорость рекомбинации должна превысить МТН. Из-за их дисбаланса начнут уменьшаться: $f_{c}$, а значит, и $\gamma$, и возрастание скорости рекомбинации. Появление на дне зоны проводимости дополнительных незаполненных состояний приведет к тому, что возрастание МТН усилится. Это усиление роста МТН, с учетом уменышения роста скорости рекомбинации, приведет к возрастанию: $f_{c}$, инверсии населенности и коэффициента $\gamma$ от уровня $\gamma_{i-m}$ до $\gamma_{L}$. В итоге этого опять усилится рост скорости рекомбинации. На графике $d^{2} I_{\mathrm{ph}} / d t^{2}=f(t)$, сделанном для части фронта $s$-компоненты, ступенька, на которую направлена стрелка, не противоречит предположенным особенностям изменения скорости рекомбинации (их проявление на крутом фронте хронограммы заметить трудно) (см. вставку на рис. 1). Возрастание $\gamma$ при $g$-переходе происходит на величину $1 / \tau_{r-m}$, характеризующую МТН, что говорит в пользу изложенного предположения.

Эмпирическое выражение (11) не только описывает изменение дифференциального коэффициента $\gamma$ усиления энергии со временем в режиме насыщения усиления собственного стимулированного излучения $\mathrm{GaAs}$ (в режиме-2). Это выражение, учитывая $\gamma \sim f_{c}-f_{v}$ [3], также отображает уменьшение инверсии населенности со временем в режиме-2. Такое уменьшение качественно подобно представленному в работе [9] в разделе „Эффект насыщения“" на рис. 40.3 для двухуровневой системы. Таким образом, $g$-переход представляется „переключением“ от режима-1 к режиму-2. В режиме-2 скорость вынужденной рекомбинации больше скорости МТН, и инверсия населенности уменьшается со временем, тогда как в режиме-1, в первом приближении, скорости равны и инверсия неизменна.

В наших условиях изменение $\gamma_{i-m}$ с $\hbar \omega$ обусловлено преимущественно изменением комбинированной, или объединенной, плотности состояний $\rho \sim\left(\hbar \omega-E_{g}\right)^{1 / 2}$, т.е. плотности вышеуказанных пар одночастичных квантовых состояний в паре $c v$-уровней, где $E_{g}-$ ширина запрещенной зоны $[10,11]$. Естественно, что, во-первых, МТН тем больше, чем больше плотность $\rho$, которой пропорционален $\gamma_{i-m}$. Отсюда следует, что МТН тем больше, чем больше $\gamma_{i-m}$. Во-вторых, чтобы превысить МТН, нужна рекомбинация с превышающей его скоростью. Эта скорость пропорциональна $E_{s}$ (в согласии с [4]), и во время $g$-перехода она пропорциональн $E_{\mathrm{th}}$. Вкупе из первого и второго следует, что увеличение $\gamma_{i-m}$ должно вызывать увеличение $E_{\mathrm{th}}$. Это качественно поясняет зависимость $E_{\text {th }}$ от $\gamma_{i-m}$ в $(6)$.

Прежде чем продолжить обсуждение поясним физический смысл времени $\tau_{r-m}$. В работе [12] было получено аналитическое выражение характерного времени $\tau_{T}$ остывания оптически накачанных носителей заряда в GaAs. Учитывалось, что остывание происходило в присутствии стимулированного излучения GaAs. Взаимодействие излучения с носителями, оно очевидно из пояснений к формуле (12), замедляло остывание накачанных носителей и соответственно ЭТН. При интенсивном $s$-излучении плотность и температура неравновесных носителей заряда становятся взаимосвязаны. Благодаря этому становятся тоже взаимосвязаны время $\tau_{T}$ и характерное время $\tau_{r-\Sigma}$ релаксации: (а) плотности носителей; (б) интенсивности $s$-излучения, интегрального по спектру, вышедшего из гетероструктуры; (в) скорости вынужденной рекомбинации носителей [4],

$$
\tau_{r-\Sigma} \approx(2 / 3) \tau_{T} \approx(2 / 3)\left(A+B \tau_{p} T_{c}^{1 / 2} E_{g}\right) \tau_{h},
$$

где $A=6.3, B=0.36, \tau_{h} \approx 0.8$ пс - время релаксации энергии электронно-дырочной плазмы (ЭДП) за счет эмиссии оптических фононов с учетом разогрева последних [13], $\tau_{T}, \tau_{p}, \tau_{h}$ измеряются в пс, $T_{c}$ - температура ЭДП в $\mathrm{K}, E_{g}$ - в эВ. Первое слагаемое в правой части выражения (12) учитывает разогрев ЭДП, связанный с тем, что энергия носителей заряда, участвующих в стимулированной рекомбинации, меньше средней энергии носителей в ЭДП. Время $\tau_{p}-$ это характерное время движения фотона излучения в активной среде, 
пока фотон не покинет эту среду или не будет поглощен:

$$
\tau_{p}^{-1} \approx c \cdot \chi^{-1}\left(\beta+D^{-1}\right) .
$$

Здесь $\beta=\sigma n-$ коэффициент поглощения света свободными носителями заряда, $\sigma=1.5 \cdot 10^{-17} \mathrm{~cm}^{2}[14]$, $n=p-$ концентрация ЭДП, $D-$ диаметр активной области. Время $\tau_{T}$ по заключению [5] имеет более широкое значение, чем считалось изначально. Оно характеризует энергетическую релаксацию носителей (следовательно, и ЭТН) с момента их накачки.

Аналитическое выражение времени $\tau_{T}$ было получено в теории [12] для случая, когда излучение одномодовое. Но в работе [4] подтвердилось предположение о том, что это выражение можно распространить и на случай, когда излучение является световым континуумом, как в GaAs. Подтверждением было то, что выражению (12) удовлетворяла экспериментальная зависимость $\tau_{r-\Sigma}(D)$. К этой последней зависимости впоследствии оказались близки зависимости $\tau_{r-m}(L)$. Это позволяет предположить следующее. Поскольку время $\tau_{T}$, а значит, и время $\tau_{r-\Sigma}$ характеризует ЭТН, то время $\tau_{r-m}$ характеризует МТН. Тогда той $s$-компоненте, у которой $\tau_{r-m}$ меньше, соответствует бо́льший $\mathrm{MTH}$, а значит, бо́льшая $E_{\mathrm{th}}$. Это соответствует зависимости (6). В целом присутствие $\gamma_{i-m}$ и $\tau_{r-m}$ в (6) означает, что плотность энергии $E_{\text {th }}$ тем больше, чем соответственно: (a) больше плотность состояний, на которые МТН доставляет носители; (б) интенсивнее доставляющий эти носители МТН.

Выше мы обсудили, насколько возможно, физический смысл полученных эмпирических закономерностей.

\section{6. Заключение}

В настоящей работе обнаружено условие перехода от экспоненциального к линейному возрастанию со временем плотности энергии спектральной компоненты собственного стимулированного излучения тонкого слоя GaAs. Подразумевается плотность энергии в активной среде. Это же условие, естественно, справедливо для интенсивности $I_{\mathrm{ph}}$ компоненты вышедшего из гетероструктуры излучения. Переход происходит, когда произведение коэффициента ненасыщенного усиления $\gamma_{i-m}$ на время, в течение которого идет экспоненциальное усиление, достигает экспериментально установленной константы. Это универсальное условие не противоречит аналогичному условию, предсказанному ранее теоретически для усиления в пространстве (его экспериментальные подтверждения нам не известны).

Та плотность энергии компоненты, при которой происходит переход от экспоненциального к линейному возрастанию, оказалась эмпирической функцией коэффициента $\gamma_{i-m}$ и характерного времени $\tau_{r-m}$ релаксации компоненты. Время $\tau_{r-m}$ тем больше, чем медленнее транспорт в энергетическом пространстве носителей заряда на энергетические уровни, с которых носители вынужденно рекомбинируют. Для компоненты также эмпирически получено представление об уменьшении со временем дифференциального коэффициента усиления $\gamma$ и инверсии населенности. Это уменьшение происходит на фронте компоненты при насыщении усиления.

С учетом предыдущих исследований получается, что все обнаруженные характерные свойства энергии $E_{s}$ и ее плотности, а также интенсивности $I_{\mathrm{ph}}$ каждой компоненты определяют два ее параметра: $\gamma_{i-m}$ и $\tau_{r-m}$. По сути это означает зависимость от: (а) вероятности тех вынужденных межзонных переходов электронов, которые увеличивают $E_{s}$ и $I_{\mathrm{ph}}$ и могут уменьшать инверсию населенности; (б) энергетического транспорта носителей, создающего и „питающего“ инверсию населенности, но замедленного из-за взаимодействия носителей с излучением. Уточним, что для представления эволюции компоненты важно еще и следующее. Скорость спонтанной рекомбинации приобретает значение, соответствующее плотности накачиваемых носителей $n=p>1 \cdot 10^{18} \mathrm{~cm}^{-3}$, с инерционностью не более нескольких пикосекунд. Благодаря этому в наших экспериментах спонтанное излучение и его усиление, создающие стимулированное излучение, возникают не медленнее, чем за пикосекунды.

Завершая, перечислим те наблюдавшиеся нами явления в режиме насыщения усиления собственного стимулированного излучения GaAs, которые, по-видимому, впервые экспериментально подтверждены или обнаружены. Это целесообразно сделать для того, чтобы способствовать корректированию и дополнению представления о насыщении усиления в полупроводнике типа $\mathrm{A}^{\mathrm{III}} \mathrm{B}^{\mathrm{V}}$.

1. Создание излучением провала в спектре усиления.

2. Взаимосвязь температуры и плотности ЭДП.

3. Терагерцовая автомодуляция населенности носителей заряда (при достаточно высоком качестве гетероструктуры) в поле излучения, создающая соответственно модуляцию фундаментального поглощения света и модуляцию излучения.

4. Связь характерного пикосекундного времени релаксации плотности ЭДП и интенсивности собственного излучения, интегрального по спектру, с характерным временем остывания носителей заряда.

5. Антикорреляция между максимальной интенсивностью этого излучения и характерным временем остывания носителей заряда.

6. Превышение длительности излучения, интегрального по спектру, над длительностью пикосекундной накачки и особенности формы огибающей излучения, вызванные замедлением энергетического транспорта носителей заряда.

7. Зависимость характерного пикосекундного времени релаксации спектральной компоненты излучения от длины усиления компоненты, или диаметра ее активной области.

8. Переход от экспоненциального к линейному усилению во времени (или от режима ненасыщенного усиления к режиму насыщения усиления) при выполнении 
условия, универсального для спектральных компонент излучения.

9. Влияние замедления транспорта неравновесных носителей в энергетическом пространстве на максимальную плотность энергии спектральной компоненты в активной среде, время достижения этого максимума и длительность компоненты.

10. Стремление к универсальному минимальному остаточному характерному времени релаксации компоненты при приближении к нулю диаметра ее активной области.

11. Уменьшение дифференциального коэффициента усиления компоненты, соответственно и инверсии населенности, при линейном возрастании интенсивности компоненты.

Большинство закономерностей в перечисленных явлениях аппроксимируются эмпирическими формулами, доказывающими существенную роль энергетического транспорта носителей, замедленного из-за их взаимодействия с излучением. Развитие теоретических исследований энергетического транспорта носителей заряда, происходящего в полупроводнике во время собственного стимулированного излучения и влияющего на излучение, теоретический вывод части названных выше явлений, продвинули бы теорию насыщения усиления. Тем более, что последняя является одной из областей недостаточно исследованной нелинейной динамики генерации стимулированного излучения в прямозонных полупроводниках.

\section{Финансирование работы}

Работа выполнена в рамках государственного задания.

\section{Конфликт интересов}

Авторы заявляют, что у них нет конфликта интересов.

\section{Список литературы}

[1] Н.Н. Агеева, И.Л. Броневой, Д.Н. Забегаев, А.Н. Кривоносов. ФТП, 55, 434 (2021).

[2] L.W. Casperson. J. Appl. Phys., 48, 256, (1977).

[3] В.Д. Соловьев. Физика лазеров. Текст лекций (4-й курс). http://elib.spbstu.ru/d1/2313.pdf/download/2313.pdf

[4] Н.Н. Агеева, И.Л. Броневой, Д.Н. Забегаев, А.Н. Кривоносов. ФТП, 53, 1471 (2019).

[5] Н.Н. Агеева, И.Л. Броневой, Д.Н. Забегаев, А.Н. Кривоносов. ФТП, 56 (3), 307 (2022).

[6] Н.Н. Агеева, И.Л. Броневой, Д.Н. Забегаев, А.Н. Кривоносов. ЖЭТФ, 143, 634 (2013).

[7] Н.Н. Агеева, И.Л. Броневой, Д.Н. Забегаев, А.Н. Кривоносов. ФТП, 54, 1018 (2020).

[8] Н.Н. Агеева, И.Л. Броневой, А.Н. Кривоносов, С.Е. Кумеков, С.В. Стеганцов. ФТП, 36, 144 (2002).

[9] Г.С. Ландсберг. Оптика (М., Физматлит, 2003).

[10] В.Л. Бонч-Бруевич, С.Г. Калашников. Физика полупроводников (М., Наука, 1990).
[11] О. Звелто. Принципы лазеров (СПб., Лань, 2008).

[12] Ю.Д. Калафати, В.А. Кокин. ЖЭТФ, 99, 1793 (1991).

[13] С.Е. Кумеков, В.И. Перель. ЖЭТФ, 94, 346 (1988).

[14] J.S. Blakemore. J. Appl. Phys., 53, R123 (1982).

Редактор Г.А. Оганесян

\section{Transition from an exponential to a linear increase of the energy density of the spectral component of picosecond stimulated GaAs emission upon gain saturation}

\author{
N.N. Ageeva, I.L. Bronevoi, D.N. Zabegaev, \\ A.N. Krivonosov \\ Kotel'nikov Institute \\ of Radioengeneering and Electronics, \\ Russian Academy of Sciences, \\ 125009 Moscow, Russia
}

\begin{abstract}
At the beginning of high-power optical picosecond pumping of the GaAs layer of the $\mathrm{Al}_{x} \mathrm{Ga}_{1-x} \mathrm{As}-\mathrm{GaAs}-\mathrm{Al}_{x} \mathrm{Ga}_{1-x} \mathrm{As}$ heterostructure, stimulated picosecond emission appears in it. The increase with time in the energy density of each spectral component of the emission occurs exponentially until the onset of the gain saturation regime. In this regime, the increase is already linear. In the present work, it is experimentally determined, depending on which parameters of the spectral component of the emission and according to what law, the following changes: (a) the time (counted from the beginning of the stimulated emission) after which the transition from exponential growth to linear one occurs; (b) the energy density of the component at the ,moment" of the transition; (c) gain at the stage of linear increase in the component energy density. The Conclusion summarizes phenomena found in our works, occurring during the gain saturation.
\end{abstract}

